\title{
Ambiances
}

anbiances Environnement sensible, architecture et espace urbain Varia | 2015

\section{Ambiances et civilité}

A propos de la contribution goffmanienne aux études sur les ambiances Ambiances and CivilityAbout the Goffmanian Contribution to the Ambiances Studies

\section{Anthony Pecqueux}

\section{OpenEdition}

\section{Journals}

Édition électronique

URL : http://journals.openedition.org/ambiances/580

DOI : 10.4000/ambiances.580

ISSN : 2266-839X

Éditeur :

Direction Générale des Patrimoines - DAPA - MCC, UMR 1563 - Ambiances Architectures Urbanités $(\mathrm{AAU})$

Référence électronique

Anthony Pecqueux, «Ambiances et civilité », Ambiances [En ligne], Varia, mis en ligne le 30 juillet 2015, consulté le 03 mai 2019. URL : http://journals.openedition.org/ambiances/580 ; DOI : 10.4000/ ambiances.580

Ce document a été généré automatiquement le 3 mai 2019.

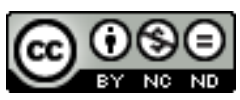

Ambiances is licensed under a Creative Commons Attribution-NonCommercial-NoDerivatives 4.0 International License. 


\title{
Ambiances et civilité
}

\author{
A propos de la contribution goffmanienne aux études sur les ambiances \\ Ambiances and CivilityAbout the Goffmanian Contribution to the Ambiances \\ Studies
}

Anthony Pecqueux

1 Ce texte ${ }^{1}$ prend prétexte de la récente édition en français d'un grand livre de sociologie urbaine pour aborder quelques liens possibles entre la micro-sociologie de l'interaction et les études sur les ambiances: Behavior in Public Places - Comment se comporter dans les lieux publics (Goffman, 2013). En effet, la nouvelle initiative par Daniel Cefaï de traduction de textes fondateurs des sciences sociales prive les chercheurs francophones sur l'espace public urbain d'une de leurs dernières possibilités de distinction: citer Goffman en anglais ${ }^{2}$. Ce geste du dernier chic est désormais obsolète tant la traduction proposée est des plus pertinentes, jusque dans l'ironie mordante du titre qui rappelle les manuels de savoir-vivre dont Goffman nourrit son texte (How to... ?, par exemple : How to Be a Lady ?). Alors même que cette ironie, présente chez le "pendant philosophique " ${ }^{3}$ de Goffman, John L. Austin, n'avait précisément pas su être rendue dans la traduction française de How to do Things with Words: Quand dire, c'est faire (Austin, 1970).

2 De plus, l'imposante postface du traducteur (Cefaï, 2013) fournit à la fois une contextualisation érudite bienvenue, et une hypothèse de lecture de la place du livre des plus stimulantes : non seulement sa place dans l'œuvre de Goffman mais aussi sa postérité dans les études urbaines, essentiellement nord-américaines et francophones. La piste proposée par Cefaï a trait à la portée politique de Goffman, non tant au sens d'une microphysique du pouvoir foucaldienne, qu'à celui d'une "interrogation sur les fondements interactionnels et situationnels de la res publica» (p. 243). La première dimension est présente dans la mesure où, au-delà de ses multiples commentaires sur la race, la classe et le genre, Goffman se livre à un « catalogue de relations observables entre inférieurs et supérieurs [...], par exemple dans la non réciprocité de l'attention donnée et reçue » (p. 253). La seconde dimension, centrale pour Cefaï, s'appuie sur un postulat fort : «Les propriétés situationnelles ne sont ainsi pas seulement des invariants anthropologiques, mais 
des gonds civils qui articulent l'ordre public » (p. 262 ; il souligne), c'est-à-dire qui rétablissent le lien civil, assurent au moins paix et sécurité. C'est toute l'originalité de sa lecture qui complète celle déjà décisive en la matière de Jean-Samuel Bordreuil (2007) ; j’y reviendrai.

$\mathrm{BPP}^{4}$ pose en 1963 une pierre essentielle à ce qu'Isaac Joseph identifie pour la seconde moitié du $\mathrm{XX}^{\mathrm{e}}$ siècle comme «le passage d'une problématique macrosociologique du malaise dans la civilisation à la problématique microsociologique du malaise dans l'interaction" (Joseph, 1984, p. 66). Ce livre s'inscrit dans une période charnière de l'œuvre de Goffman, particulièrement productive: il est écrit quatre ans après $L a$ présentation de soi, deux ans après Encounters et Asiles, et la même année que Stigmates... Il s'appuie sur trois principaux types de données ethnographiques: le travail d'enquête pour sa thèse dans les îles Shetland (et autres saynètes captables dans l'espace public); une observation participante menée à ce qu'il appelle "l'Hôpital Central ", un hôpital psychiatrique de Washington qui fournit l'assise empirique d'Asiles; et des données de seconde main, issues de manuels de civilité et de coupures de presse, voire d'extraits de romans, etc.

Le présent article se déploie en trois mouvements: le premier retrace les principaux apports de BPP, notamment du point de vue de l'attention sensible et de son organisation en situation. Le deuxième mouvement suit le fil de quelques pistes de BPP en direction des ambiances urbaines, comprises comme la tonalité affective dans laquelle les individus interagissent et qu'ils peuvent modifier par leurs interactions et présences. Enfin, le dernier mouvement s'appuie sur les séries télévisées The Wire et Treme pour illustrer et poursuivre la discussion sur ambiances et civilité entamée dans la section précédente. C'est dire que cet article commence comme un compte-rendu de BPP, pour progressivement en dégager des hypothèses de lecture qui seront mises à l'épreuve de ces séries télévisées. La première de ces hypothèses est que ce livre de Goffman offre des pistes puissantes pour lier les questions de perception et d'organisation de l'attention à des questions politiques comme celle de la civilité (et donc ne pas cantonner les premières, comme c'est souvent le cas, à une sphère neutre, apolitique). La seconde hypothèse poursuit un travail précédent (Pecqueux, 2012), à savoir sur un fond inextricablement politique des ambiances, lié à leur dimension temporelle (de durabilité) et qui serait saisissable ici à travers la conception goffmanienne de la civilité, qui ne se réduit pas à des paroles ou des actes mais s'étend également à l'atmosphère qui enveloppe les situations et interactions. Selon cette hypothèse, la civilité n'est pas que " sociale " (en un sens étroit) mais aussi spatiale et temporelle. La mise en évidence de cette dernière dimension est aussi une manière de renouveler la lecture de Goffman (et plus généralement de l'interactionnisme) en relativisant une de ses critiques habituelles, à savoir son présentisme.

\section{Une sociologie de la vie publique focalisée sur l'attention}

BPP est une enquête sur les "formes élémentaires de conduite " (p. 6) dans les lieux publics (voire semi-publics), à partir de l'hypothèse que la focalisation sur ce qui y est approprié ou non est susceptible d'informer plus largement sur l'organisation sociale et sa normativité, plus spécifiquement sur l'ordre de l'interaction - cette question qui taraude Goffman jusqu'à ses derniers mots (Goffman, 1988). La systématisation de cette hypothèse tout du long conduit à l'idée que chaque situation de coprésence, chaque 
rassemblement forme «un petit dépôt de l'organisation sociale» (p. 205). Une telle problématique le conduit à débusquer les règles des rassemblements mais aussi toutes les dérogations aux règles, qu'elles soient voulues ou non, comme les transgressions ou les ratés de l'étranger, ainsi que des "personnes rituellement à part » comme les jeunes enfants ou les personnes âgées (p. 104)5 ${ }^{5}$, évidemment les fous, etc. : c'est-à-dire toutes les personnes vis-à-vis desquelles nous ne formons pas les mêmes attentes normatives du fait précisément de leur statut.

6 Une autre façon de le dire : BPP décrit nombre de nos manières de faire pour « respecter l'esprit ou l'ethos de la situation » (p. 12), ainsi que les conséquences occasionnées toutes les fois où cet esprit n'est pas respecté, avec en ligne de mire la conséquence ultime de ce non-respect : le risque d'internement (p. 208). C'est là l'intérêt et l'originalité de Goffman, son ambiguïté encore, et c'est dans cette perspective qu'il faut lire cette quasi-clôture du livre :

«Un rassemblement social, même lâchement défini, reste une pièce exigüe : il présente plus de portes permettant d'en sortir et plus de raisons normales, d'un point de vue psychologique, de vouloir les franchir que ne peuvent l'imaginer ceux dont la loyauté à la société situationnelle est inébranlable » (p. 203).

7 La normativité qui s'en dégage n'est pas liée à un automatisme (déterminisme) d'un système dual règles / sanctions. Elle est plus fine, réglée sur la seule nécessité de prendre en compte les règles : soit pour s'y conformer, soit pour les contourner - dans les deux cas de figure de quantité de manières différentes (de manière habile, subtile, provocatrice, etc., p. 39).

Cela l'amène à pouvoir établir de manière assez précise ce que l'individu des classes moyennes occidentales ${ }^{6}$ peut faire pour se présenter comme « une personne apprêtée pour l'interaction sociale» (p. 166), principalement par la «modulation appropriée de ses engagements situés» (p. 167). En réalisant ces ajustements, il marque une forme de respect pour l'occasion sociale. Il montre qu'il sait «s'acquitter de ce qu['il] doit à l'occasion sociale » (p. 166), ou qu'il en est - momentanément, durablement, sciemment... - incapable.

9 Sur cette base générale, fidèle en cela à la "tradition goffmanienne " (sa marque de fabrique au sein de la littérature socio-anthropologique), BPP fournit une myriade de définitions et d'outils de première importance, entrecoupés de données ethnographiques souvent croustillantes. S'enchaînent ainsi les définitions et différences entre rassemblement, situation et occasion (p. 18); ou entre "simplement-situé " et situationnel (p. 21-22). Ces définitions, fondées sur des données ethnographiques, sont elles-mêmes souvent hautes en couleurs: "Le risque encouru par mon propre corps quand il est braqué par un pistolet dans un vol à main armé est situationnel; la perte de mes biens domestiques est simplement-située » (p. 22). La distinction suivante entre interaction focalisée et interaction non focalisée (p. 24), i.e. entre l'interaction explicite à plusieurs et le simple coup d'œil furtif porté à l'autre, laisse transparaitre sa lecture de la sociologie des sens de Simmel, qu'il cite longuement à ce titre (p. 81-82). Cela donne le tempo de la suite : à savoir l'importance dans ce livre du sensible, avec une prédominance de l'œil (Breviglieri, Stavo-Debauge, 2007). Ainsi, les problématiques portées par BPP sont traitées à travers le prisme premier de l'attention perceptive ; en découle une focalisation sur tout ce qui est " 'à portée' » sensorielle (p. 17), qu'Isaac Joseph thématise comme la question urbaine d'un " pluralisme des portées ", où portée désigne la " capacité de charge ou la distance à laquelle porte une chose » (Joseph, 2007, p. 445). 


\section{L'organisation de l'attention}

10 A partir de ce foisonnement initial, Goffman se recentre progressivement autour des questions d'engagement dans les situations (p. 34sqq), qu'il traite d'abord à travers les manifestations de l'attention, dans la mesure où s'engager (plus ou moins) nécessite une (gestion de son) absorption dans l'activité. Et dans la mesure également où la vie sociale à laquelle nous n'échappons pas implique précisément des formes d'engagement dans les situations au sein desquelles nous sommes embarqués. Dans ce cadre, Goffman introduit une notion particulièrement importante, celle de "tonus interactionnel ", qui s'oppose aussi bien à l'« irrespect " qu'à l'« engourdissement interactionnel» (p. 24-28). Prises ensemble, ces notions dessinent une échelle des modes de présence aux situations. L'aspect de tonus interactionnel, que Goffman développe plus avant dans "Fun in games $»^{7}$, a trait à une bonne gestion de l'apparence personnelle (le facework ou travail de la face) par une forme de vigilance : une « vivacité et (...) sensibilité à la situation » (p. 24). L'attention quasi-obsessionnelle des hommes à vérifier en public si leur braguette de pantalon est bien fermée (p. 25) est un exemple de ce tonus. Il montre que parfois «la force provient moins des muscles que de la classe sociale » (p. 134), c'est-à-dire l'idée que ce n'est pas seulement pour un soulagement physiologique (se gratter, se moucher, aller aux toilettes...) que l'on se permet des latéralités ou distractions au sein d'un engagement attentionnel intense: c'est aussi socialement qu'on ne peut être le spectateur et/ou participant imperturbablement attentif souvent présumé.

11 Le quatrième chapitre, intitulé "Quelques règles à propos de la distribution de l'engagement » (p. 40sqq), est déterminant dans la mesure où il reproblématise (à la suite notamment des premiers sociologues urbains de Chicago, au premier rang desquels Robert E. Park) la dimension perceptive de la participation à la situation, qui est d'abord affaire d'attention et d'organisation de l'attention - notamment sa manifestation (ou dissimulation) aux co-présents. Goffman distingue dans un premier temps engagement principal et engagement secondaire, qui introduisent ce qu'on désigne communément désormais sous le terme de "multi-activité » (multitasking), à savoir la capacité à faire plusieurs choses en même temps, en prêtant plus ou moins d'attention à ces différentes choses simultanées. Dans un second temps, il affine cette distinction par celle entre engagement dominant et engagement subordonné, qui distribue socialement ces «plus ou moins ": les situations et les statuts des individus portent avec eux leur lot d'obligations qui amènent à adopter tel engagement comme dominant au détriment d'autres (relégués alors comme subordonnés).

En pratique, ces engagements sont susceptibles de se superposer dans une joyeuse indétermination qui montre encore la finesse des ajustements que les individus réalisent (ou ratent) en situation. Par exemple: il arrive que nous poursuivions un objectif qui n'implique pas un engagement principal ; c'est le cas à chaque fois que nous empruntons les transports en commun: le but est d'atteindre notre destination, mais il n'y a guère d'activité principale associée à ce but. Nous sommes alors nombreux à compenser cette absence grâce à des "sources transportables d'engagement» (p. 46) : journaux, livres, tricots, mais aussi désormais téléphones portables, lecteurs mp3, etc. Bien avant le Mobility Turn des sciences sociales (Sheller \& Urry, 2006), Goffman décrit nos manières de ne pas nous contenter du paradigme du déplacement (la mobilité comme moyen pour la seule fin de se rendre d'un point $\mathrm{A}$ à un point $\mathrm{B}$ ), de réaliser pratiquement le paradigme 
de la mobilité par laquelle la mobilité peut être une fin en soi, notamment par les activités que les agents y mènent.

Sans détailler l'ensemble des cas de figure abordés par Goffman, il ne saurait être question pour autant de passer sous silence la présence d'une notion centrale de son œuvre: l'inattention civile, et son articulation avec la question de l'organisation de l'attention en public (p. 73sqq). Cette notion, retravaillée dans Les relations en public (Goffman, 1973), abondamment commentée depuis (entre autres : Quéré \& Brezger, 1993), est un principe de «politesse minimale» (p. 75) entre étrangers dans l'espace public; il garantit à chacun un droit à l'indifférence. Ce " plus infime des rituels interpersonnels (... ) qui pourtant régule constamment le commerce social entre les personnes dans notre société » (p. 74) correspond à la forme élémentaire de coordination qu'accomplissent des passants qui se croisent, et par laquelle chacun reste à sa place (ne serait-ce que pour éviter une collision) et reconnaît à l'autre une place, sa place ni plus ni moins : celle d'une personne, qui marche d'un certain côté de la chaussée. Il s'agit d'un savoir pratique des justes distances qui combine la reconnaissance de la présence d'autrui et l'absence d'empiètement de son territoire. L'inattention civile consiste donc à «traiter les personnes présentes du seul point de vue de leur participation à un rassemblement, en mettant entre parenthèses leurs autres caractéristiques sociales » (p. 75).

L'analyse de la temporalité du coup d'œil par David Sudnow (2002) en est un exemple ordinaire : le coup d'œil doit être assez long pour montrer qu'on saisit l'autre comme une personne, et assez court pour manifester qu'on ne cherche pas à en savoir plus à son sujet. C'est là la juste distance : ni trop ni pas assez. Dans ce cadre général, il s'agit alors tout autant de ne pas empiéter visuellement sur le territoire d'autrui (par un regard prolongé), que d'essayer de ne pas attirer l'attention, de tenter de passer pour commun ou ordinaire : de "faire 'être comme tout le monde' (Sacks, 2002). Ainsi, l'inattention civile remplit une fonction essentielle pour l'organisation sociale : elle permet à chacun de s'assurer des intentions non belliqueuses d'autrui à son égard, et de lui montrer que les siennes ne le sont pas non plus. Bref: attester d'une publicité pacifique, au sein de laquelle chacun «n'a rien à craindre à voir ou à être vu » (p. 74 ; voir aussi n. 1 p. 115). Le droit à l'indifférence qui en émerge est également éloigné du mépris perceptif, qui traite l'autre (le domestique, le noir...) comme s'il n'existait pas, comme une «non personne » (p. 73-74), comme s'il était littéralement invisible ${ }^{8}$. Il s'agit in fine tout autant d'un droit à la différence, dans la mesure où celle-ci reste « convenable » : s'exprime dans des limites congruentes avec les exigences de la situation.

\section{Civilité ordinaire et tonalité affective d'une situation}

Cette caractérisation perceptive de faits sociaux aussi élémentaires et importants, et plus largement la problématisation en termes d'organisation de l'attention pour élaborer une anthropologie de la vie publique, commencent à décliner la portée de la sociologie goffmanienne pour les ambiances. Avant de systématiser cette exploration, constatons l'intérêt pluriel de Goffman pour les ambiances et du point de vue des études sur les ambiances. Déjà, du fait de sa préoccupation répétée pour la notion d'ethos, qu'il définit en toute généralité comme une "atmosphère de groupe » (p. 85), et qu'il emprunte aux travaux de Gregory Bateson. Pour l'anthropologue américain, l'ethos correspond à la «tonalité affective générale» d'une culture (Bateson, 1986, p. 159sqq) ${ }^{9}$ : «l'élément sensible et émotif » (Ibid., p. 40) si souvent négligé mais si déterminant pour comprendre 
une culture. Goffman en cite en note le passage central, qui exemplifie la notion par la description d'un groupe de jeunes intellectuels tenant ensemble une conversation sur un mode cynique: "il s'établit entre eux une ambiance et un type de comportement particuliers qui indiquent un ethos" (Bateson, op.cit., p. 160). Et déjà, comme chez Goffman, la meilleure démonstration est celle réalisée en creux par le gaffeur : celui qui rate le mode cynique et fait une remarque sérieuse - il ne respecte donc pas l'ethos de la situation.

Cette idée qu'il est possible de caractériser l'ambiance des interactions et qu'elle est déterminante pour comprendre l'ordre de l'interaction n'est qu'une première facette de l'intérêt de BPP pour les ambiances. La lecture proposée ci-dessous déploie cet intérêt à un double niveau; sur un premier plan, cela a trait au caractère perceptif de l'engagement dans une situation par l'organisation de l'attention, cette fois à partir des figures du body torque et de la dérive. Sur un second plan, consécutif : quand il n'est plus seulement question de choisir entre des engagements possibles, ou de définir perceptivement la situation, mais que l'enjeu se déplace vers la focalisation de l'attention et/ou l'action dans la situation et selon la situation (sa tonalité affective). Je l'aborderai avec les figures de la civilité ordinaire comme ouverture et de ce que je désigne comme la paradoxale liberté ambiantale des classes populaires. Cela dessine un lien pluriel et réciproque entre ambiances et interactions : la tonalité affective d'une situation dirigeant les interactions dans une certaine mesure, et inversement, les interactions (également, les caractéristiques des individus: leur appartenance sociale, etc.) pouvant modifier la tonalité affective d'une situation. Cela oriente également du côté d'une lecture politique des ambiances.

\section{D'une tonalité affective à l'autre}

17 Dans son projet global de «parler d'interactions en termes d'ambiances» (Bordreuil, 2013, p. 142), Bordreuil note que Goffman «intercal[e] entre acteurs et 'Umwelt' le filtre intermédiaire des 'involvements' interactionnels » (p. 143 ; il souligne). Cela signifie bien que la notion d'engagement est une des manifestations de la qualité de l'expérience (cette relation entre un organisme et un environnement selon John Dewey - par exemple: 1993) ; or ces éléments ne sont pas fixes mais pris dans une dynamique constante (James, 2007). C'est pourquoi les agents sont susceptibles de passer d'une situation à l'autre par un ajustement des engagements, et ainsi de passer d'une tonalité affective à l'autre. Ce processus est décrit par l'ethnométhodologue américain Emanuel Schegloff (1998) comme un body torque.

Cela caractérise un moment où un individu est engagé dans plus d'une activité, et le manifeste par une direction corporelle divergente entre l'orientation du cou et celle de la taille, qui ne se maintient pas durablement: le corps se rassemblant en direction de l'engagement choisi (soit par un retour à la position de départ, soit par l'adoption d'une nouvelle position). Schegloff se réfère souvent à Goffman et surtout à BPP, essentiellement pour la distinction entre engagement dominant et subordonné. Ce livre analyse effectivement plusieurs types de situations évoquant le body torque. Cela transparaît déjà pour ce qu'il appelle le « dépêtrement ultime » (p. 125), à savoir accorder une attention à contrecœur (alors qu'on est déjà engagé dans une autre activité), y répondre de manière évasive pour ensuite se dégager de cette attention - ce qui signifie 
un retour manifeste à la position de départ. Goffman prend l'exemple parlant de la sollicitation par un enfant d'un parent affairé.

Cela devient d'autant plus saillant avec les «désaffections tout à fait mineures et momentanées » (p. 152-153) que nous ne sommes pas capables de ne pas avoir lors d'engagements intenses ${ }^{10}$. Ces désaffections forment une situation de body torque dans la mesure où elles induisent de se focaliser sur autre chose que sur l'engagement présent. Si elles se prolongent, elles peuvent devenir des signes et symptômes de maladie mentale ${ }^{11}$; il cite ainsi le cas de cette patiente d'une institution psychiatrique qui joue au ping-pong tout en cherchant à suivre la partie de bridge de la table voisine (p. 153). Ces différentes illustrations se rapprochant du body torque dressent une certaine conception de l'individu en public (en société), à savoir un individu «écartelé, mais écartelé sur un chevalet standard, dressé sur un mode standard » (p. 134).

Parmi les travaux sur les modes de présence des individus aux situations, ceux d'Albert Piette, du Mode mineur de la réalité (1992) à ses derniers développements (par exemple: Piette, 2014), ont contribué à prolonger la perspective interactionniste en lui conférant une dimension temporelle, souvent manquante. Par exemple en traquant non seulement les modes mineurs d'engagement des individus dans les situations (e.g. un rituel catholique), mais également en les suivant avant et après leur participation à un événement, ce qui permet de replacer ces engagements dans une chaîne temporelle plus longue. C'est dans cette perspective qu'il faut comprendre la citation par Piette de cette critique d'une application trop « stricte » de l'interactionnisme, de la part d'un de ses plus éminents représentants actuels, Jack Katz :

«À travers l'étude des comportements dans les lieux publics, les avancées commencées par Georg Simmel et prolongées par Erving Goffman et John Lofland n'ont pas connu de nouvelles impulsions depuis trente ans. Il est temps de quitter la perspective atemporelle et instantanée de l'observateur limité à un espace, pour insérer le sens de la situation en cours dans le cadre temporel élargi de la biographie d'un participant. Au fur et à mesure que le chercheur se déplace d'une scène d'interaction à une autre, il sait ce que les autres membres de la situation ne peuvent connaitre pleinement, que ce qui est en train de se passer comporte des enjeux associés au passé et au futur, ainsi qu'à l'ensemble des trajectoires d'une vie » (Katz, 2009, p. 286, cité et traduit par Piette, 2014, p. 23) ${ }^{12}$.

Dans ce cadre, s'il paraît essentiel d'introduire de la temporalité par le suivi des individus, un des intérêts des travaux sur les ambiances est de faire porter l'accent sur la spatialisation des pratiques, et de potentiellement adjoindre des questions de temporalité, appliquées non aux seuls individus mais étendues aux lieux eux-mêmes. En effet, employer la terminologie des ambiances implique un questionnement sur la durabilité : comment même parler d'ambiance si cette dernière ne persiste pas dans le temps ou ne se répète pas régulièrement, etc. (Pecqueux, 2012) ? De telles perspectives font immanquablement penser aux travaux classiques de William $\mathrm{H}$. Whyte, par exemple sur les plazas new-yorkaises (Whyte, 1980 ; voir Brayer, 2013 et plus largement Brayer, 2014 pour une réflexion originale sur le lien entre ces travaux et les études sur les ambiances). Si une manière de prolonger l'interactionnisme consiste à suivre les biographies des individus, les travaux sur les ambiances permettraient de faire de même pour les biographies des lieux ; il y aurait ainsi tout intérêt à croiser ces deux types d'investigations temporelles - j'y reviendrai.

Pour revenir à BPP et à la discussion entamée à partir du body torque, le caractère ordinaire mais non neutre du passage d'une tonalité affective à l'autre y est prolongé par 
une autre figure: la dérive (p. $147 \mathrm{sqq}$ ). Le propos de Goffman est le suivant: si une rencontre n'est pas totalement indépendante de l'ethos ou de la «tonalité affective » de l'occasion sociale dans laquelle elle se déploie, elle n'en est pas non plus totalement dépendante. D'où son intérêt pour les phénomènes de dérive :

"De même qu'une occasion sociale prise comme un tout est susceptible de dessiner un 'profil d'engagement' et d'embarquer toutes les rencontres qu'elle abrite dans une direction donnée, de même chaque rencontre particulière doit manifester des propriétés dynamiques qui lui soient propres, non seulement en engendrant un monde pour ses participants, mais en les transportant toujours plus loin en lui » (p. 147),

quitte à ce qu'ils aillent trop loin par rapport à l'occasion de leur rencontre. Pour être clair : c'est l'exemple du groupe (la rencontre) sanctionné parce qu'il plaisante trop fort à un enterrement (l'occasion sociale); ou des personnes sobres se sentant hors cadre quand elles arrivent au sein d'une soirée déjà bien arrosée (p. 148-149). En quelque sorte, une dérive souligne une forme d'échec de la membrane (supra), qui n'a pas su rappeler, maintenir le lien avec la réalité. La dérive manifeste un engagement (un tonus interactionnel) trop intense dans la rencontre, oublieux de l'occasion dans laquelle elle prend place. S'y rend saillante une disjonction entre la tonalité affective générale de l'occasion sociale et celle occasionnée par la rencontre. Se trouve alors en jeu un double niveau d'ambiance: celle de l'occasion sociale et celle de la rencontre (l'interaction proprement dite), qui ne peuvent le plus souvent être totalement disjointes ou discordantes - ou alors dont la discordance pointe des conséquences sociales déterminantes.

C'est toute « la difficulté de rester en contact avec l'occasion sociale tout en s'impliquant spontanément dans des engagements situés » (p. 149), qui peut nécessiter de se soulager par des techniques de dissimulation, notamment des «écrans de l'engagement» (p. 36-37; 149-151), comme la conversation, la voiture, etc. Ainsi commence à être posée la question de l'environnement construit, notamment quand les écrans de l'engagement fonctionnent comme des «barrières perceptives» (p. 36), derrière lesquelles il est possible de se réfugier pour échapper à l'observabilité (et ainsi réaliser des actes qui seraient sanctionnés en public). Il évoque les chambres à coucher et les salles de bain mais on pourrait prendre d'autres dispositifs architecturaux: visuels / sonores / ... Grégoire Chelkoff et Jean-Paul Thibaud (1993) en ont étudié plusieurs fondés sur un jeu de visibilisation ou dissimulation de ou dans l'espace public. Ils parlent notamment de surexposition («mettre visuellement en valeur une chose au détriment d'une autre», p.9) et de filtrage (un tamis entre deux unités distinctes ou deux milieux, p.12). Ce dernier dispositif s'apparente aux écrans de l'engagement de Goffman. Toujours est-il que par rapport au body torque, la discussion entamée à partir de la dérive ouvre sur la centralité de l'environnement construit pour les deux niveaux d'ambiance mis en évidence.

\section{La civilité ordinaire comme ouverture}

Sur un autre plan, la notion d'ambiance joue un rôle primordial dans BPP. Suivons pour cela le raisonnement de Goffman à propos de la civilité ordinaire (p. 109sqq). Il commence par analyser les "personnes ouvertes » (p. 110) : il entend par là que certaines positions sociales créent les conditions pour des interactions entre étrangers, comme les statuts de policiers ou de prêtres. Bref : toutes les personnes-ressources d'une civilité ordinaire sans 
forcément de contrepartie - on ne dit pas uniquement "bonjour» à un policier en uniforme pour lui demander son chemin, on peut aussi le faire simplement pour le saluer. Parfois ce sont des personnes qui créent ces conditions, et non un statut social dont on peut prendre congé (par exemple en enlevant son uniforme de policier); il s'agit alors de personnes ouvertes, comme le jeune enfant ou le petit vieux. A l'inverse, pour ouvrir des engagements de face avec un inconnu, soit celui-ci se trouve exposé (vulnérabilisé) par la situation, soit on est soi-même une personne ouvrante, car ouverte (p. 112).

A partir de là, Goffman étend sa réflexion aux espaces : il est alors question de régions ouvertes (p. 115sqq), c'est-à-dire des espaces d'accessibilité mutuelle. Par là, il désigne les espaces susceptibles d'occasionner des solidarités informelles, comme lors de la reconnaissance mutuelle comme membres de groupes dominés et/ou « rituellement à part ", à l'instar des noirs entre eux (p. 114). Ou alors quand il y a exposition mutuelle : deux passants qui se touchent dans la rue; ou les échanges de regards voire de gestes entre un automobiliste et un piéton pour savoir qui passera le premier; etc. Quand ils permettent des engagements de face entre étrangers, les espaces sont appelés des régions ouvertes. De telles régions dressent les contours d'ambiances «bonnes» pour l'interaction car basées sur la "bienveillance et [la] réciprocité des égards » (p. 118) qui, ensemble, garantissent "accessibilité et sociabilité » entre étrangers. Les interactions susceptibles d'émerger passent le plus souvent par une conversation phatique, destinée à garantir des intentions mutuelles pacifiques des inconnus (p. 120).

Si le regard du sociologue tend a priori à privilégier les villages et les villes modestes comme terrains privilégiés de cette civilité ordinaire (p. 116), pour l'interconnaissance qu'ils occasionnent, des configurations urbaines l'affordent également. Goffman évoque les espaces exigus comme les ascenseurs (p. 118-119), mais aussi les différentes formes de bars, les moments de carnaval (Agier, 2000, p. 34sqq) comme de festival (Bordreuil, 2013). Il faut aussi compter, en creux, avec toutes les ouvertures illégitimes, telles celles exercées de manière sensible (sifflements, interpellations, regards insistants...) sur les femmes dans l'espace public (p. 124). Ces ouvertures illégitimes forment, avec le mépris perceptif, une sorte d'envers de l'inattention civile : comme une attention barbare - en tout cas un usage barbare, dé-civilisé, des sens. Elles peuvent occasionner en retour des parades, comme quand le port des oreillettes par des femmes (même sans musique) vise à manifester une non-disponibilité : à faire rater l'ouverture illégitime par incapacité pour l'ouvreur de statuer sur la perception ou non de l'ouverture. Les oreillettes fonctionnent alors comme un écran de l'engagement.

Dans sa postface, Daniel Cefaï rapproche cette question des régions ouvertes de la notion de "lieu sociopète » (p. 255-6), une sorte de «bonne ambiance " par laquelle se rendent sensibles les compétences de rassemblement des citadins (pour parler comme Isaac Joseph $)^{13}$. Cela traduit une forme d'hospitalité du lieu dans la mesure où il remplit les conditions de "félicité de la coprésence entre étrangers» (p. 256): sans idéalisme ni angélisme, seulement parce qu'il rend possible «l'inattention civile qui assure la coexistence pacifique ». Mais aussi parce que de tels lieux possèdent certaines caractéristiques construites: ils "sont facilement accessibles, procurent un plaisir esthétique et une aise pratique, ne sont ni déserts ni bondés, présentent de bonnes conditions d'éclairage et de son " (Ibid.; on peut renvoyer à nouveau, sur cette question, aux travaux de W.H. Whyte sur les plazas). En somme, les lieux sociopètes, au premier rang desquels figure un espace public urbain réussi, dessinent les conditions de l'expérience publique, à savoir sa publicité, son accessibilité et sa visibilité. 

caractéristiques d'une région ouverte ou d'un lieu sociopète peuvent être rapprochées des environnements décrits par Joëlle Zask (2011) comme facilitateurs d'une expérience démocratique pleine - donc participative, mais dans une certaine acception du terme. Elle examine d'abord les notions bergsoniennes de sociétés ouverte et fermée ( Ibid., p. 118sqq). Une société ouverte est « favorable à l'intuition, à l'imagination créatrice, à l'élan vital ou à l'impulsion [...] gouvernée par la démocratie [...] dynamique » (Ibid., p. 119). Mais une telle société est forcément éphémère, seulement «temporairement ouverte»: "L'ouverture est exceptionnelle. Elle résulte d'une action spéciale suffisamment forte pour provoquer une perturbation» (p. 122). Par là se confirmerait l'hypothèse formulée ci-dessus sur la nécessaire durabilité d'une ambiance, pour qu'il soit même possible de parler d'ambiance. Cela ouvre également sur le questionnement politique qui en découle, à savoir sur les conditions à remplir (notamment en termes de perturbation) pour qu'une ambiance puisse figurer au rang des environnements susceptibles d'occasionner une pleine expérience démocratique.

Il semblerait de ce point de vue qu'il faille d'une part revoir les ambitions à la baisse, et que pour cela, d'autre part, les perspectives de Goffman sur les régions ouvertes et leur prolongement en lieu sociopète par Cefaï gagneraient à être revisitées à partir de celles de Donald Winnicott sur les environnements facilitateurs (vs. défaillants) (Zask, op.cit., p. 237sqq). Au sein de ce nouveau couple oppositionnel, un environnement facilitateur est décrit par Winnicott comme "'suffisamment bon' [...] 'convenable' ». Il n'a donc pas l'exceptionnalité de la société ouverte, il correspond «simplement » à l'environnement susceptible de permettre à l'enfant de développer son existence, de s'individuer (ce qui n'est pas toujours simple non plus...). Un tel environnement émerge à partir d'un nœud de relations entre l'organisme et l'environnement, ce qui rapproche de la définition de l'expérience de John Dewey, spécialement quand l'expérience est symbiotique.

\section{La paradoxale liberté ambiantale des classes populaires}

31 Sur un dernier plan, BPP ouvrirait des hypothèses nouvelles quant aux ambiances, notamment si l'on continue à s'intéresser aux questions de civilité. Elles commencent par la description d'un parc urbain « un samedi après-midi, en été » (p. 169). Cette ambiance particulière autorise un certain relâchement de la gestion de soi et corrélativement des objets de l'engagement: "la 'présence' situationnelle est réduite au minimum. Tel individu peut défaire ostensiblement son nœud de cravate, enlever ses chaussures, et être moins soucieux d'étouffer des renvois » (Ibid.). Cela renvoie à la description par Jacques Cheyronnaud des cafés-concerts du XIX ̀̀ siècle comme ces

« établissement[s] où l'on peut garder son chapeau sur la tête, fumer, boire, discuter entre soi assis autour d'une table, regarder la salle en même temps qu'une scène et, notamment, sinon écouter 'chansonnettes' ou monologues, du moins percevoir de loin, parmi tant de distracteurs (discussions, apostrophes, bris de verres, etc.), chanter ou déclamer... » (Cheyronnaud, 2012).

Il s'agit du constat classique, joliment formulé dans les deux cas, que certaines occasions sociales et leurs espaces associés favorisent des ambiances de relâchement par rapport aux normes de comportement d'une société.

Goffman le note, de telles ambiances varient d'une culture à l'autre, voire à l'intérieur même des cultures (p. 170). En entrant plus avant dans la granularité : figurent encore des variations selon les lieux et les moments, par exemple entre les différents bâtiments de 
l'hôpital psychiatrique dans lequel il a enquêté (p. 172). Enfin, il existe encore des différences pour les ambiances selon les genres et selon les rôles (p. 173-174), avec à l'extrémité d'une échelle le patient chronique qui prend le rôle de « dingue » et se soucie peu de son apparence publique, et à l'autre extrémité les "dignitaires de haut rang », qui portent souvent l'uniforme de leur rôle, avec ce à quoi il oblige pour les co-présents comme pour eux.

Par conséquent, à partir de la description d'un parc urbain l'été, et en suivant le fil des ambiances de relâchement, Goffman en arrive à proposer une hypothèse forte, guère testée à ma connaissance: celle d'une meilleure adhérence des classes populaires ${ }^{14} \grave{a}$ l'environnement physique, construit, comparativement aux classes moyennes et donc supérieures. "Ceux qui travaillent sans cravate, et n'ont pas à se soucier de ne pas salir leurs vêtements, peuvent se permettre de toucher et d'être touchés par leur environnement physique » (p. 173) ; par exemple, de "s'appuyer contre un mur» ou d'utiliser n'importe quel substitut de siège pour s'asseoir. A contrario, pour les classes moyennes et supérieures, «le fait que leurs modèles de vêtements et de propreté soient incompatibles avec une trop grande familiarité avec l'environnement physique de la rue semble être autant la cause que l'effet de leur discipline situationnelle» (p. 173). Bien entendu, il ne saurait être seulement question de salir ou non ses vêtements; l'enjeu concerne plus généralement les postures corporelles, les manières de vivre l'environnement.

35 Cela dit, et cette seconde hypothèse - interdépendante de la première - rend le propos plus complexe et nuancé qu'il n'y paraît : cela ne signifie pas forcément une plus grande liberté pour les classes populaires. Ou alors une liberté paradoxale: celle d'être tenu simultanément à être plus vigilant vis-à-vis de l'environnement social.

" Dans les situations où un individu est supposé montrer beaucoup de respect pour le rassemblement comme tel, il peut être exempté de toute sorte d'engagement en profondeur avec ceux qui se trouvent à proximité. Quand tous les coups sont permis, par contre, une lutte interpersonnelle, extrêmement prenante et usante, risque de s'ensuivre » (p. 177).

Pour ceux qui peuvent se permettre de relâcher leurs attitudes et attentions (engagements) en public, le prix à payer est susceptible de devenir parfois important voire exorbitant car il peut leur être nécessaire de «rester mentalement sur leurs gardes » (p. 177), de ne pas « lever [leurs] défenses » (p. 168). Il n'est pas sûr que Goffman formulerait en de tels termes cette hypothèse d'une paradoxale liberté ambiantale des classes populaires, c'est-à-dire d'un relâchement à l'égard de l'environnement construit contrebalancé par une vigilance accrue vis-à-vis de l'environnement social. Il s'agit bien tout à la fois d'une piste de lecture, et d'une hypothèse de travail qui aurait à être mise à l'épreuve.

En forme d'illustration de cette liberté paradoxale (c'est encore une lecture libre du passage), Goffman réalise un développement original sur le bruit, qui ne devient offense ("source de trouble») que dans la mesure où il s'apparente à un «sur-engagement en situation » (p. 181). A l'instar du jeune homme interné pour « avoir mis le volume de son phonographe trop fort au YMCA »: non tant parce que les employés se seraient sentis offensés, mais plutôt parce qu'ils ne pouvaient comprendre le "monde que le jeune homme habite et qu'il remplit en le saturant de musique » (p. 181). Ce jeune homme a pris la liberté d'occuper musicalement l'espace de l'auberge de jeunesse mais s'est exposé ainsi à en subir les conséquences, que Goffman analyse moins comme une riposte face à 
une provocation initiale que comme une réaction face à l'anormalité d'un comportement et son incompréhensibilité ${ }^{15}$. En somme, ce jeune homme a pris des libertés avec son environnement, mais il n'en est pas quitte pour autant; et ce sont bien différentes facettes de l'environnement (construit ou spatial / social) qui entrent là en tension.

\section{« Un nouveau Baltimore »?}

Les pistes de travail sur ambiances et interactions tirées de BPP ont en commun d'orienter vers une lecture politique des ambiances - en un certain sens de politique. En effet, Goffman se préoccupe des ambiances essentiellement dans la mesure où elles peuvent (ou non) être caractérisées comme «bonnes pour l'interaction»: lui offrir des points d'appui, d'encouragement, ou encore la miner, l'empêcher, la contraindre, etc. Cela se ressent pour les formes les plus élémentaires du type de la civilité ordinaire, comme pour des formes plus explicites comme les lieux sociopètes. A chaque fois, il y est question d'un élément aussi difficilement saisissable que les ambiances, à savoir le fondement sensible du lien civil, comme le souligne Cefaï à propos de la civilité et de ses échanges de politesse minimale: «Ce sont ces multiples obligations et responsabilités, éprouvées par tout un chacun dans son commerce avec les autres, qui tissent le lien civil, dans sa texture sensible et pratique la plus ordinaire » (Cefaï, 2013, p. 289) ${ }^{16}$. En ce sens, la civilité pour Goffman n'est pas seulement affaire de «mœurs", de souci ou politesse envers autrui; elle est, également, indissolublement liée à l'environnement et aux exigences de la situation. Elle n'est pas que sociale, mais également spatiale.

Si comme on l'a vu, chaque rassemblement forme « un petit dépôt de l'organisation sociale » (loc.cit.), et si les ambiances constituent la tonalité affective générale de chaque rassemblement, elles contribueraient alors à l'ordre social, et la civilité pourrait en être une exemplification. C'est en tout cas la pente qui va désormais être suivie, à partir du matériau empirique que constituent les séries télévisées créées par David Simon pour la chaîne HBO : The Wire surtout (Sur écoute, 2002-2008, portrait noir en cinq tableauxsaisons de la ville de Baltimore ; Simon, 2011), mais aussi Treme (2010-2013, chronique en quatre saisons de la vie quotidienne à La Nouvelle-Orléans post-Katrina; Simon \& Overmyer, 2014). Si elles ont été récemment abondamment commentées par les sciences sociales de l'urbain (voir notamment Aarons, Burdeau, Chamayou et al., 2011, Bacqué, Flamand, Paquet-Deyris et al., 2014) ${ }^{17}$ - au point de devenir parfois une sorte de passage obligé d'un contemporanéisme chic-, BPP donne des pistes pour en proposer une lecture renouvelée. Surtout, dans la lignée des pistes dégagées, de telles séries offrent une occasion assez unique pour se plonger dans les dimensions temporelles et spatiales propres aux ambiances d'un lieu (ici, à chaque fois, une ville promue au rang de personnage principal), et y adjoindre les biographies (le suivi temporel) des individus qui y prennent part. En cela, elles permettraient de tirer les leçons tant d'un interactionnisme temporalisé, que de l'intérêt de la prise en compte des interactions sociales pour les travaux sur les ambiances, et bien sûr du croisement heuristique de tous ces éléments.

40 Si la première saison de The Wire se focalise sur les trafiquants de drogue qui irriguent Baltimore, la seconde évoque principalement la désindustrialisation qui frappe son port ; la troisième, une forme d'utopie (hétérotopie) urbaine qui relègue la drogue à distance ; la quatrième, les affres du système éducatif américain; et la dernière, le pouvoir de la 
presse (David Simon fut journaliste au Baltimore Sun - son principal co-scénariste, Ed Burns, étant pour sa part un ancien inspecteur de la brigade criminelle de Baltimore).

Par rapport aux thématiques dégagées dans BPP, The Wire illustrerait déjà en partie la paradoxale liberté ambiantale des classes populaires à travers le relâchement postural des dealers dans l'espace public urbain. Ces types «à la cool » par excellence des quartiers populaires des villes américaines contemporaines se reconnaissent par leurs postures relâchées : par exemple leurs positions assises (vautrées) sur un canapé au milieu d'un espace vert au pied des projects (saison une); ou encore sur les marches des entrées d'immeuble, ou debout au coin de deux rues, etc. Mais ces postures relâchées ont un prix, celui d'être en même temps l'objet de tous les regards (et de toutes les écoutes) : ceux de leurs clients à l'affût d'un possible réapprovisionnement ; et surtout ceux des enquêteurs de police, tout aussi à l'affût, d'une possible arrestation cette fois ${ }^{18}$.

Je vais me concentrer sur un aspect précis de la série, à la charnière entre les saisons trois et quatre - celle consacrée à une forme d'utopie reléguant la drogue loin de la vie urbaine et celle posant la question du système éducatif. En effet, la saison trois s'attaque à un problème classique des métropoles post-industrielles : que faire des quartiers populaires, abandonnés pendant des décennies et dont on récolte aujourd'hui les conséquences directes, à savoir une situation sociale très largement dégradée, surtout pour une jeunesse sans perspectives d'emploi ? Une première réponse consiste à mettre cet échec sur le compte de la forme architecturale : démolir celle-ci pour en proposer une nouvelle. Une seconde réponse fait porter le chapeau à la forme sociale: il s'agirait alors de redéployer les habitants dans la ville, en promouvant par exemple la mixité sociale. Une troisième solution émerge au cours de cette saison sous la houlette d'un major de police (Colvin) qui, pour faire face à la hausse sans précédent de la criminalité dans son district (ouest) et au désespoir tout à la fois de ses troupes et des citoyens du district, fait le pari de créer Hamsterdam ${ }^{19}$ : un supermarché de la drogue à ciel ouvert, sur lequel la police ferme les yeux, et contenu au sein de quelques pâtés d'immeubles désaffectés en périphérie du district. Créée à l'insu des responsables policiers et politiques de Baltimore, cette forme d'hétérotopie urbaine finira dans un scandale et un fiasco retentissants, qui contribueront grandement à coûter sa réélection au maire de la ville.

\section{Hamsterdam : vers une nouvelle civilité ?}

43 Le parti pris ici est que la saison trois vaut moins pour cette utopie ratée d'Hamsterdam, qui finit mal et que le major Colvin lui-même reconnait comme un échec (ou une fausse bonne solution); que pour le renouveau des relations sociales - de la vie urbaine - que la création d'Hamsterdam occasionne directement : la drogue étant reléguée en périphérie, les autres quartiers du district peuvent retrouver une vie urbaine civile, «normale ». Une forme de civilité élémentaire y refleurit, principalement entre ces personnes ouvertes et ouvrantes que sont les policiers "de base " (qui arpentent les rues en uniforme) et le reste de la population. Cette troisième solution vis-à-vis des quartiers populaires délaissés refuse à la fois la démolition de l'environnement construit et le changement de population, au profit de la création des conditions pour un retour de la civilité ordinaire quitte à le faire au prix d'une part d'ombre importante, celle d'Hamsterdam. L'un des principaux héros de la série, Jimmy McNulty, un inspecteur jusqu'au-boutiste dans ses enquêtes criminelles, s'engage résolument du côté de cette solution ; à savoir : il disparaît quasiment de l'écran tout au long de la saison quatre au cours de laquelle il choisit de 
redevenir un policier de base ouvrant et ouvert dans la rue, disant bonjour aux passants ce qui entraîne le meurtre d'un des dealers à qui il dira bonjour de manière trop prononcée.

La saison trois s'ouvre en effet sur la mise en scène de la démolition d'une des tours de Baltimore, précédée d'un discours du maire, Clarence Royce, alors au début d'une campagne décisive pour la primaire démocrate en vue de l'investiture aux élections municipales - au cours de laquelle il fait principalement face à des chiffres désastreux de la délinquance et à un jeune loup blanc de la politique locale (Tommy Carcetti), conseiller municipal chargé des dossiers concernant la sécurité. Au pied de la tour prête à exploser, Royce formule quelques promesses quasi-électorales puis apostrophe la foule, en faisant de la démolition à venir un symbole du renouveau de la ville : «Prêts pour un nouveau Baltimore ?! » Il actualise ainsi la première solution évoquée à propos de l'action sur les quartiers populaires délaissés, celle qui démolit et oublie et les habitants et leur mémoire, puisque les jeunes dealers qui déambulent dans le quartier avant la démolition programmée devisent sur leurs souvenirs liés à cette tour (lieu de leurs premiers émois sexuels, etc.).

Le passage central pour mon propos a lieu à partir du milieu du onzième (pénultième) épisode de la saison trois: quand le major Colvin, débusqué dans son initiative d'Hamsterdam et débarqué de la police pour cela, est sollicité par le conseiller municipal Carcetti pour qu'il lui donne des explications sur cette hétérotopie urbaine et ses effets à peine croyables - la baisse vertigineuse des chiffres de la délinquance dans son district. Colvin commence par l'y emmener pour lui faire constater que les coins de rue autrefois les plus dangereux sont devenus des havres de normalité civile - pour preuve, on voit en toile de fond de leur conversation un policier dire bonjour et converser avec des passants... Ensuite, Colvin conduit Carcetti à la même réunion de concertation locale à laquelle il assistait impuissant en ouverture de saison; cette fois, l'assemblée est beaucoup plus fournie (une cinquantaine d'habitants), les chiffres de la délinquance beaucoup plus positifs et les débats beaucoup moins vindicatifs. Une africaine-américaine d'environ 40 ans prend la parole pour constater l'évolution récente au regard de son enfance dans le même quartier :

[dans son enfance] On connaissait la police, on avait un policier blanc, la maison était sur son chemin, il allait à pied et bien tous les soirs il s'arrêtait pour causer un moment avec ma mère, il s'asseyait sous la véranda pour causer. Et bien j'vais vous dire : c't homme-là son nom c'était Frazy O'Larry, il appelait même ma mère par son prénom

[Le jeune policier africain-américain en uniforme responsable de la réunion] _ Oui oui, revenons à ce qui a motivé notre réunion

_ J'vous d'mande pardon monsieur ! Excusez-moi mais j'ai pas terminé! Voyez-vous $\mathrm{y}$ a quelque chose que j'voudrais dire : j'avais pas r'vu ce face à face avec la police depuis très, très longtemps, depuis beaucoup trop longtemps jusqu'à la s'maine dernière. Un jeune officier, un officier noir est passé d'vant chez moi, j'étais sous ma véranda, il m'a donné sa carte. Il s'appelle Regie Ballard, c'est son nom, oui [en sortant et lisant ladite carte]. Il s'est assis et il a causé avec moi. On a juste causé ! Et comme ça maintenant j'connais son nom et sa tête et lui, il connaît mon nom et ma tête. Alors laissez-moi vous dire une chose : c'est ça des relations normales !

Ces derniers mots, prononcés dans un tonnerre d'applaudissements, sont du pain béni pour Colvin et sa démonstration en direction de Carcetti. Il n'a pas même besoin de les commenter: ils illustrent d'eux-mêmes les effets recherchés par Colvin à travers la création saugrenue d'Hamsterdam (bref: sa part de félicité), à savoir rétablir par un 
« compromis citoyen» (loc.cit.) une urbanité normale - donc civile - dans le reste du district. Après cette démonstration, Colvin ne cache pas l'envers de la médaille au conseiller municipal: il lui montre ce qui l'a rendu précisément possible, à savoir Hamsterdam, part sordide d'une civilité retrouvée.

Dans l'épisode suivant, le dernier de la saison, McNulty parle à plusieurs reprises de ses projets encore flous de changement de vie, mais les situe : «Dans le district ouest, à l'ouest je me sens chez moi ». Ce n'est qu'en clôture d'épisode qu'il les concrétise: il renfile l'uniforme de policier de base, puis on le voit déambuler dans la rue en riant avec les passants. La saison quatre ne nous montre donc que par intermittences cet anti-héros qu'est devenu McNulty, arpentant à découvert (en uniforme) les rues qu'il surplombait auparavant de ses investigations camouflées (des écoutes plus ou moins légales des dealers et des prises photographiques depuis des toits d'immeubles). Ce nouveau rôle le fait quasiment disparaître de l'écran, puisqu'à ces enquêtes spectaculaires il troque des bonjours aux passants et qu'à ses beuveries nocturnes quotidiennes avec son collègue Bunk se substitue une vie de famille modèle. Ces nouveaux ressorts n'ont a priori pas l'intensité dramatique requise pour une telle série, qui poursuit son investigation des basfonds de Baltimore. Pour autant, McNulty apparaît de temps à autres, notamment dans une scène centrale de l'épisode d'ouverture de la quatrième saison (voir l'annexe pour sa transcription), au cours de laquelle l'inspecteur Carver tente d'insuffler à son co-équipier (manifestement peu réceptif...) sa nouvelle façon d'envisager la guerre à la drogue, qui passe par une connaissance fine du terrain, donc par une connaissance personnelle des dealers voire par des liens avec certains d'entre eux. Dans ce cadre, il n'est pas anodin que cela s'actualise par des échanges de politesse, certes un peu forcés. Car il n'est plus question de simples citoyens qui se reconnaissent dans la rue, pas plus que des relations civiles anonymes possibles entre les passants et les personnes ouvrantes et ouvertes que forment les policiers en uniforme. Il s'agit cette fois des relations quasi-impossibles entre inspecteurs de police et dealers en exercice.

On voit les ressources que constituent de telles séries: on entre dans l'épaisseur spatiale et temporelle des lieux, ainsi dans celle des individus qui les peuplent. Suivre McNulty ou suivre les souvenirs d'enfance d'une Africaine-américaine anonyme contribuent au même résultat.

\section{La civilité ordinaire : (in)félicités}

Dans le douzième (pénultième) épisode de la saison quatre, les téléspectateurs sont à nouveau confrontés à la civilité ordinaire, cette fois de manière définitivement tragique puisque des échanges de bonjour entre l'agent de terrain McNulty et le dealer Bodie y sont directement présentés comme la cause de l'exécution de ce dernier par la bande de Marlo, dominant le district. Le lendemain, quand McNulty apprend la mort de Bodie, il fait semblant d'arrêter un gars de son coin de rue et lui demande, après l'avoir plaqué face contre le mur, qui a tué Bodie :

Vous tous! Ils l'ont cramé parce que vous êtes flic et qu'il vous parlait. On dit qu'on l'a vu avec un flic devant le dépôt. Alors coffrez-moi ou laissez-moi dégager de ce coin de rue avant qu'il m'arrive la même chose !

Ce passage pointe la fin et l'échec de la civilité à Baltimore : quand on est policier, y dire bonjour en public à un dealer signerait en quelque sorte l'arrêt de mort de ce dernier. $\mathrm{Si}$ Marlo suspectait d'être balancé par Bodie, il voulait avant tout délivrer un «message » 
par son exécution ; ce message, du point de vue des petits dealers de coin de rue comme celui alpagué par McNulty, est bien plus large : pour être exécuté par un homme de main de Marlo, il n'est pas nécessaire de balancer, il suffit de "parler » à un policier - d'être civil en somme. Cela peut être croisé avec l'analyse des conditions de félicité du performatif par John L. Austin (1970) : le langage peut être performatif - agir - seulement dans certaines circonstances (conditions sociales). Dire «je vous marie» accomplit le mariage si et seulement si vous êtes maire ou habilité à marier; si les deux personnes à marier correspondent aux standards légaux en vigueur (un homme et une femme, dans la plupart des pays); si cela est réalisé en un certain lieu à une certaine heure; etc. De la même manière, la civilité ordinaire est perçue comme telle (comme une manifestation positive de socialité) si et seulement si les personnes échangeant des politesses ne se trouvent pas chacune d'un côté d'une frontière symbolique infranchissable - en l'occurrence, dealers vs. policiers. A la limite, on est là face à une règle ancestrale du crime organisé; or, les codes imposés dans le district ouest par la bande de Marlo l'outrepassent largement en multipliant de façon exponentielle ces frontières symboliques, jusqu'à confiner à l'empêchement de toute civilité. En l'occurrence : si vous ne voulez pas finir exécuté dans une maison abandonnée de Baltimore, vous ne devez pas trop parler à certaines filles du quartier, vous ne devez pas non plus trop vous rapprocher de votre professeur de maths qui a repéré vos talents, ni vous acoquiner avec un gangster homosexuel qui joue au Robin des Bois du ghetto ; etc.

51 Après cet échec cuisant (avoir provoqué la mort de Bodie), McNulty annonce à sa compagne qu'il va quitter l'uniforme de policier de base pour rejoindre l'enquête sur les corps des maisons abandonnés en tant qu'inspecteur, ne serait-ce que pour honorer la mémoire de Bodie. Il lui en indique aussi les conséquences inévitables: il sera alors différent, moins bien, il va boire à nouveau, voir des filles... En reprenant l'uniforme pour arpenter les rues et leur vie quotidienne afin de contribuer à y rétablir la civilité, McNulty s'associait (plus ou moins consciemment) à un projet plus global concernant un renouvellement des ambiances de Baltimore et passant par les interactions ordinaires ; en le quittant définitivement pour enfiler à nouveau les habits civils d'inspecteur de la brigade criminelle, tout en ayant conscience des conséquences néfastes pour sa santé et celle de son couple, il entérine le fiasco de ce projet, qui a achoppé sur la possibilité même qu'un policier et un dealer se parlent.

Au bout de ce parcours sur les saisons trois et quatre de The Wire, on est manifestement loin d'un "nouveau Baltimore » au sens d'un « meilleur » Baltimore, d'un Baltimore plus civil, aux rues et ambiances apaisées, qu'on a pu entrevoir à la fin de la saison trois et espérer voir durer pendant la saison quatre, avec McNulty en gardien ordinaire du temple de la civilité. Cette dernière y apparaît comme un effet d'Hamsterdam, qui est annihilé une fois qu'Hamsterdam est rasé (en une nuit!) ; cet effet connaît une simple hysteresis entre la fin de la saison trois et quelques séquences du début de la saison quatre. Du coup, la question émerge : la civilité ordinaire dans une métropole comme Baltimore ne seraitelle actuellement qu'une chimère d'un passé révolu ou le seul effet bénéfique d'un monde rendu aseptisé de force (dont la gangrène serait reléguée loin, ailleurs) ${ }^{20}$ ? Il faut sans doute comprendre la civilité comme située entre ces deux extrêmes; pour cela, l'autre série créée par David Simon pour HBO, Treme, est d'une aide précieuse. Elle fonctionne en toute généralité à la fois comme le complément de The Wire et comme son contrepied car elle ne prend plus comme personnages centraux des hommes publics (un inspecteur de la criminelle, un dealer, un candidat démocrate aux municipales...) mais prélève quelques 
échantillons dans la vie ordinaire (des musiciens des rues, un universitaire et sa fille, une restauratrice qui ne peut payer ses factures et son second, etc.), qu'elle juxtapose avec des figurants issus de la «vraie» Nouvelle-Orléans. Treme n'est pas non plus un portrait idéalisé de La Nouvelle-Orléans post-Katrina (où le quartier de Treme s'opposerait trait pour trait au district ouest de Baltimore) - pas plus que The Wire ne dépeignait de manière trop noire la vie du ghetto et des institutions de Baltimore.

Pour cela, Treme prend plusieurs partis - outre le choix particulier des personnages -, dont celui de l'échelle d'un quartier (nommé, jusqu'à donner son titre à la série) vs. l'impersonnalité du district (dont la dénomination ne renvoie qu'à un découpage administratif - «ouest»). D'un quartier dont les passants ne se connaissent pas tous personnellement mais se reconnaissent dans la rue. D'un quartier avec un patrimoine vivant (se renouvelant à chaque Mardi-Gras et autres fêtes traditionnelles), et fondé sur la valorisation de l'expression artistique (non spectacularisée comme dans la plupart des métropoles contemporaines et leur « quartier des spectacles » bon chic bon genre), même si elle a le plus souvent du mal à joindre les deux bouts; etc. Autant de facettes qui rendent la vie urbaine un peu moins intolérable, un peu plus supportable, un peu plus passible ; autant de facettes d'une civilité ordinaire dans l'espace public qui, à distance du «fantasme de consensus rationnel » (Cefaï, 2013, p. 287), est affaire moins d'interactions focalisées que « d'engagements latéraux, labiles et vulnérables. Liberté, égalité, solidarité y prennent un autre sens » (Ibid.). Autant de facettes d'une civilité non extraordinaire, construite sur une contribution réciproque entre ambiances et interactions, comme le suggère Goffman. En somme, de ce point de vue, The Wire et Treme nous font faire l'expérience de la puissance potentielle de la civilité, et sa potentielle puissance ambiantale car sensible comme le souligne Cefaï à propos du lien civil et de «sa texture sensible et pratique la plus ordinaire " (loc.cit.). Et elles le font en pointant l'importance de la temporalité pour saisir les effets croisés des interactions et des ambiances.

\section{Pour conclure}

Pour conclure en toute généralité sur BPP, son projet global peut être qualifié comme celui d'une esthétique sociale, à savoir l'étude des «manifestations sensibles du social, [de] la dimension esthétique de la société » (Carnevali, 2013, p. 30 ; elle souligne). En effet, le livre remplit ce projet en analysant d'un côté l'organisation de l'attention, et de l'autre la maîtrise (ou non) de ses propres apparences sensibles - à l'instar du vêtement auquel échoit souvent le «fardeau d'exprimer la bonne orientation à adopter à l'intérieur de la situation» (p. 180). Dans une perspective d'esthétique sociale, les apparences sensibles sont à la racine de toute relation sociale, non comme pathologie du social (dans une configuration notionnelle qui opposerait apparaitre et être), mais comme sa physiologie essentielle. On est loin alors d'une interprétation classique mais faible de Goffman, essentiellement fondée sur la métaphore théâtrale comme fin en soi de sa sociologie ${ }^{21}$, en faisant un chantre de «l'authenticité perdue ».

Par rapport aux intrications possibles entre la microsociologie de l'interaction et les études sur les ambiances, BPP offre des pistes inédites pour une lecture directement politique des ambiances, au sens du fondement sensible et donc ambiantal du lien civil ordinaire. Ainsi, pour dépasser la seule question de l'inattention civile, au même titre que Carole Gayet-Viaud propose de s'intéresser de près à des figures qui n'y sont pas soumises de la même manière (le bébé et le petit vieux) afin d'y débusquer «un espace 
d'élaboration et d'accomplissement des formes premières, élémentaires de l'êtreensemble " (Gayet-Viaud, op.cit., p. 66), l'analyse des ambiances sous l'angle microsociologique (et réciproquement) pourrait ouvrir des pistes nouvelles. En effet, leurs qualités dessinent quelques unes des conditions de félicité ou d'infélicité de la civilité ordinaire. Et dans ce cadre, The Wire comme Treme contribuent à augmenter la compréhension morale (voir Laugier (dir.), 2010 sur le perfectionnisme moral) de la complexité des configurations urbaines qui favorisent ou empêchent (réduisent, etc.) la civilité.

\section{BIBLIOGRAPHIE}

Aarons, Kieran ; Burdeau, Emmanuel ; Chamayou, Grégoire ; Mangeot, Philippe ; PotteBonneville, Mathieu ; Samocki, Jean-Marie \& Vieillescazes, Nicolas. 2011. The Wire, reconstitution collective. Paris : Capricci / Les prairies ordinaires.

Agier, Michel. 2000. Anthropologie du carnaval. La ville, la fête et l'Afrique à Bahia. Marseille: Ed. Parenthèses.

Austin, John L. 1970. Quand dire c'est faire. Paris : Seuil.

Bacqué, Marie-Hélène ; Flamand, Amélie ; Paquet-Deyris, Anne-Marie \& Talpin, Julien (eds.). 2014. The Wire. L'Amérique sur écoute. Paris : La Découverte.

Bateson, Gregory. 1986. La cérémonie du Naven. Paris : Le livre de poche.

Berger, Mathieu; Cefaï, Daniel \& Gayet-Viaud, Carole (eds.). 2011. Du civil au politique.

Ethnographies du vivre-ensemble. Bruxelles: Peter Lang.

Bidet, Alexandra ; Boutet, Manuel ; Chave, Frédérique ; Gayet-Viaud, Carole \& Le Méner, Erwan. 2015. Publicité, sollicitation, intervention. Sociologies [En ligne], Dossiers, Pragmatisme et sciences sociales : explorations, enquêtes, expérimentations. Disponible sur : http:// sociologies.revues.org/4941 (consulté le 02 mars 2015).

Bonicco-Donato, Céline. 2013. La métaphore théâtrale et la théorie des jeux dans l'œuvre d'Erving Goffman. Paradigmes individualistes ou situationnistes? In : Cefaï, Daniel ; Perreau, Laurent (eds.). Erving Goffman et l'ordre de l'interaction. Paris : PUF / CURRAP / CEMS. p. 267-285.

Bordreuil, Jean-Samuel. 2007. Isaac Joseph : la politique depuis Goffman. In : Cefaï, Daniel ; Saturno, Carole (eds.). Itinéraires d'un pragmatiste. Autour d'Isaac Joseph. Paris : Economica. p. 101-134.

Bordreuil, Jean-Samuel. 2013. Ambiances, partage d'ambiances et prisme interactionnel. In : Thibaud, Jean-Paul ; Duarte, Christiane Rose (eds.). Ambiances urbaines en partage. Pour une écologie sociale de la ville sensible. Genève : Metis Presses. p. 139-154.

Brayer, Laure. 2013. Filmer l'ambiance urbaine : Les dispositifs vidéographiques à l'œuvre chez William $\mathrm{H}$. Whyte dans La vie sociale des petits espaces urbains. Ambiances [En ligne], Représentation - Traduction - Ecriture. Disponible sur : http://ambiances.revues.org/335 (consulté le 12 février 2015). 
Brayer, Laure. 2014. Dispositifs filmiques et paysage urbain. La transformation ordinaire des lieux à travers le film. Thèse de doctorat. Grenoble : Université de Grenoble. Disponible sur : http:// hal.univ-grenoble-alpes.fr/tel-01123772 (consulté le 10 mars 2015).

Breviglieri, Marc ; Stavo-Debauge, Joan. 2007. L'hypertrophie de l'œil. Pour une anthropologie du « passant singulier qui s'aventure à découvert ». In : Cefaï, Daniel ; Saturno, Carole (eds.).

Itinéraires d'un pragmatiste op.cit. p. 79-98.

Carnevali, Barbara. 2013. L'esthétique sociale entre philosophie et sciences sociales. Tracés, Horssérie $\mathrm{n}^{\circ} 13$. p. 29-48.

Cefaï, Daniel. 2003. L'enquête de terrain. Paris : La découverte.

Cefaï, Daniel. 2010 (ed.). L'engagement ethnographique. Paris : Editions de l'EHESS.

Cefaï, Daniel. 2013. L'ordre public. Micropolitique de Goffman. In : Goffman, Erving. Comment se conduire dans les lieux publics. Notes sur l'organisation sociale des rassemblements. Paris : Economica. p. 209-290.

Cefaï, Daniel ; Perreau Laurent (eds.). 2013. Erving Goffman et l'ordre de l'interaction. Paris : PUF / CURRAP / CEMS.

Chelkoff, Grégoire ; Thibaud Jean-Paul. 1993. L'espace public, mode sensible : le regard sur la ville. Les annales de la recherche urbaine $\mathrm{n}^{\circ}$ 57-58. p. 7-16.

Cheyronnaud, Jacques. 2012. Marseille et son Alcazar. Les termes d'une doxa. Actes en ligne de la rencontre internationale Morphologie et mondes du music-hall. Marseille, 7-8 octobre 2010 : Centre Norbert Elias (EHESS - CNRS) / Hall de la Chanson. Disponible sur : http://www.lehall.com/ galerie/musichall/?p=131 (consulté le 15 janvier 2014).

Conein, Bernard. 2005. Les sens sociaux. Trois essais de sociologie cognitive. Paris : Economica.

Dewey, John. 1993. Logique. La théorie de l'enquête. Paris : PUF.

Gayet-Viaud, Carole. 2006. De l'innocuité sociale aux degrés d'humanité. Les types du petit vieux et du bébé. Les annales de la recherche urbaine $n^{\circ} 100$. p. 59-67.

Goffman, Erving. 1961. Encounters : Two Studies in the Sociology of Interaction. Indianapolis : BobbsMerrill.

Goffman, Erving. 1973. La mise en scène de la vie quotidienne, 2. Les relations en public. Paris : Ed. de Minuit.

Goffman, Erving. 1988. L'ordre de l'interaction. In : Ibid. Les moments et leurs hommes. Paris : Ed. de Minuit. p. 186-230.

Goffman, Erving. 1991. Les cadres de l'expérience. Paris : Ed. de Minuit.

Goffman, Erving. 2013. Comment se conduire dans les lieux publics. Notes sur l'organisation sociale des rassemblements. Traduit et présenté par Daniel Cefaï. Paris : Economica (« Etudes sociologiques »).

Honneth, Axel. 2005. Invisibilité : sur l'épistémologie de la « reconnaissance ». Réseaux n 129-130. p. 39-57.

James, William. 2007. Philosophie de l'expérience. Un univers pluraliste. Paris : Les empêcheurs de penser en rond.

Joseph, Isaac. 1984. Le passant considérable. Essai sur la dispersion de l'espace public. Paris : Librairie des méridiens. 
Joseph, Isaac. 2007. Pluralisme et contiguïtés. In : Ibid. L'athlète moral et l'enquêteur modeste. Paris : Economica. p. 437-459.

Katz, Jack. 2009. Time for new urban ethnographies. Ethnography. vol. 10, n² 2-3, p. 285-304.

Laugier, Sandra. 2008. L'ordinaire transatlantique. De Concord à Chicago, en passant par Oxford. L'homme n' 187-188. p. 169-199.

Laugier, Sandra. 2013. La vulnérabilité de l'ordinaire. Goffman lecteur d'Austin. In : Cefaï, Daniel ; Perreau, Laurent (eds.). Erving Goffman et l'ordre... op.cit. p. 339-368.

Laugier, Sandra (ed.). 2010. La voix et la vertu. Variétés du perfectionnisme moral. Paris : PUF. Lemieux, Cyril. 2009. Le devoir et la grâce. Pour une analyse grammaticale de l'action. Paris : Economica.

Pecqueux, Anthony. 2012. Politiques des ambiances - Que faire du fond inextricablement politique des ambiances? In : Thibaud, Jean-Paul ; Siret, Daniel (eds.). Ambiances in action / Ambiances en acte(s) - International Congress on Ambiances, Montreal 2012. Montreal : Canada. p.145-150.

Pecqueux, Anthony. 2013. Tempesta sonora o fuoco di campo sonoro. Do The Right Thing, interazione e ascolto musicale (in) publico = Sonic storm or sonic field fire: Do the Right Thing, interaction and music listening in public. Rivista Studi culturali vol. $10 \mathrm{n}^{\circ} 1$. p. 53-70.

Pharo, Patrick. 1985. Le civisme ordinaire. Paris : Librairie des méridiens / Klincksieck et Cie.

Piette, Albert. 1992. Le Mode mineur de la réalité. Paradoxes et photographies en anthropologie. Louvain : Peeters.

Piette, Albert. 2014. Les enjeux d'une anthropologie existentiale : vigilance et dissection. Recherches qualitatives. vol. 33, $\mathrm{n}^{\circ} 1, \mathrm{p} .19-40$.

Quéré, Louis ; Brezger, Dieter. 1993. L'étrangeté mutuelle des passants. Le mode de coexistence du public urbain. Les annales de la recherche urbaine $\mathrm{n}^{\circ}$ 57-58. p. 89-100.

Sacks, Harvey. 2002. Faire « être comme tout le monde ». In : Thibaud, Jean-Paul (ed.). Regards en action. Ethnométhodologie des espaces publics. Bernin : A la croisée. p. 201-210.

Schegloff, Emanuel A. 1998. Body Torque. Social Research vol. 65 n³. p. 535-596.

Sheller, Mimi ; Urry, John. 2006. The new Mobilities Paradigm. Environment and Planning A 38 (2). p. 207-226.

Simon, David. 2011. Sur écoute. L'intégrale de la série [24 DVD]. Home Box Office / Warner Bros.

Simon, David ; Overmyer, Eric. 2014. Treme. L'intégrale de la série [14 DVD]. Home Box Office / Warner Bros.

Sommer, Robert. 1967. Sociofugal space. American Journal of Sociology vol. 72 n 6. p. 654-660.

Sudnow, David. 2002. Les paramètres temporels de l'observation interpersonnelle. In : Thibaud, Jean-Paul (ed.). Regards en action op.cit. p. 57-81.

Whyte, William H. 1980. The Social Life of Small Urban Spaces. New York: Project for Public Spaces. Zask, Joëlle. 2011. Participer. Essai sur les formes démocratiques de la participation. Paris : Ed. Le bord de l'eau. 


\section{ANNEXES}

Transcription d'une scène de l'épisode d'ouverture de la quatrième saison, The Wire (Simon, 2011, S4, E1)

Bodie [un des dealers que l'on suit depuis la première saison] est à son coin de rue avec trois de ses acolytes; les inspecteurs Carver et Colicchio débarquent en trombe comme des cow-boys, laissant leur voiture quasi en travers de la route.

Carver [très théâtral tout du long] : Et les sentiments, Bodie ?!? Où sont ces putains d'sentiments ??! [à Colicchio] C'lui-là j'lui courais déjà après quand il était encore mineur, mais est-ce qu'au moins on s'en souvient ? Est-ce que j'ai droit à un sourire, un signe de tête, un salut? Rien! Tout simplement 'bonjour'...

Bodie : [de mauvais gré] Bonjour...

Carver : Tu vois ! C'est pas si difficile !

Carver s'adresse de manière ironique aux trois acolytes de Bodie ; McNulty débarque, laissant également sa voiture en travers.

McNulty : Ça va?

Carver : Ouais, on causait

(...)

McNulty [à Brodie, en quittant la scène] : Quand je repasse dans une heure ce coin est nettoyé et les gars ont fini pour aujourd'hui, ok?

Carver [après le départ de McNulty] : Bon alors on réessaye. Je dis : ‘Très bonne soirée, monsieur Brodus [le vrai nom de Bodie]'

Bodie : Et bonne soirée à vous sergent Carver

Carver [en se tapant le front] : Tu vois, ça rentre là !

Bodie : [dans un grand sourire] Et très bonne soirée aussi à vous, officier Colicchio

Colicchio [très énervé] : Tu t'la carres bien profond, tas d'merde ! [Carver et Colicchio retournent vers leur voiture ; Colicchio est toujours vexé qu'un dealer lui ait dit bonsoir] C'est quoi l'problème?

Carver: Tony, on peut pas engueuler tout le monde

Colicchio : Ah ouais?

Carver : Avec qui tu vas causer quand y aura des merdes?

\section{NOTES}

1. Il a bénéficié des remarques utiles des deux relecteurs anonymes de la revue Ambiances, ainsi que de celles des participant-e-s du séminaire « Anthropologie du 'spectaculaire' et sociologie des processus culturels » (EHESS - Marseille, mars 2014). 
2. Il reste au moins Encounters (Goffman, 1961). Il faut signaler le double contexte de cette parution: 1/ les nombreux travaux de Daniel Cefaï sur l'ethnographie et ses textes centraux (2003; 2010), et plus spécifiquement sur Goffman. Le volume collectif qu'il a récemment dirigé avec Laurent Perreau (2013) propose deux nouvelles traductions de textes de Goffman et de nombreuses recherches originales inspirées par ce dernier. 2/ La collection "Etudes sociologiques » des éditions Economica, dirigée par Jean-Philippe Heurtin et Danny Trom, qui œuvre à traduire en français des travaux classiques de sociologie pragmatique (Joseph Gusfield, Hans Joas, Niklas Luhmann, etc.), tout autant qu'à publier des études originales dans cette même «famille» sociologique. Notons par exemple, pour les questions de perception susceptibles d'intéresser les chercheurs travaillant sur les ambiances, l'important livre de Bernard Conein (2005) ainsi que la revisite sociologique des affordances par Cyril Lemieux (2009, p. 95sqq).

3. Pour les liens entre Goffman et Austin, voir Laugier, 2008, 2013.

4. Désormais, par commodité, je désigne ce livre par les initiales du titre original, Behavior in Public Places : BPP. Pour les mêmes raisons de commodité, les paginations non référées renvoient à des passages de BPP. Sauf indication contraire, les soulignements sont de Goffman.

5. Pour une réflexion sur ces deux figures, voir Gayet-Viaud, 2006.

6. Des années 1960 : inutile de préciser que pour une part, qui lui a été largement reprochée, le propos de Goffman est situé et daté ; on aura compris que ce n'est pas la pente de lecture privilégiée ici.

7. Goffman, 1961, p. 15-72 ; voir également Bordreuil, 2013, p. 142-145, qui l'aborde sous l'angle de la membrane qui assure une transduction entre l'investissement dans une situation (en l'occurrence de jeu) et le monde réel qui entoure (enserre, etc.) cette situation. En un mot : «pas de jeu sans membrane qui sépare le jeu du monde environnant»(p. 144), ce que Bordreuil précise au fur et à mesure moins comme une séparation que comme un « tamis » de la réalité (p. 145).

8. Mépris perceptif sur lequel Axel Honneth a fondé sa théorie de la reconnaissance à partir du roman de Ralph Ellison, L'homme invisible, dont le narrateur (noir) n'est tout simplement pas «vu» par les blancs qui l'entourent: il subit par là «une forme particulièrement subtile d'humiliation raciste ", par laquelle il est réduit à « une non-existence au sens social du terme » (Honneth, 2005, p. 39).

9. Plus précisément, il s'agit de l'« expression d'un système culturellement normalisé d'organisation des instincts et des émotions des individus » (Ibid., p. 159 ; il souligne).

10. Dans Les cadres de l'expérience (Goffman, 1991, p. 202-209), Goffman rethématise cette question en termes de «canal de distraction ». Il désigne par là ce qui menace notre engagement, que ce soit pour nous soulager ou nous proposer un autre engagement.

11. A nouveau, la vie publique oscille entre normalité et pathologie; le risque d'internement venant attester de sa vulnérabilité fondamentale.

12. Texte original: "In the study of behavior in public places, the advances begun by Georg Simmel and continued by Erving Goffman and John Lofland have not seen new leaps for 30 years. It is time to move beyond the atemporal, fly-on-the-wall perspective of the situationally specific participant observer to see the meaning of the current situation within the longer-term framework of a participant's biography as he or she moves from one arena of situated interaction to another, always aware of what in situ co-respondents cannot fully know, that what is currently happening has retroactive and prospective meanings based on the overarching trajectories of his or her own social life ».

13. Un lieu sociopète s'oppose à un autre dit «sociofuge». Pour nuancer cela, les travaux de Robert Sommer montrent avec acuité d'une part qu'aucune architecture (autant construite que morale) n'a pu annihiler la communication humaine (les prisons - et plus largement les institutions asilaires - en sont un bon exemple, avec leur vie communicationnelle souvent intense mais clandestine); et d'autre part qu'on peut parfois vouloir s'isoler des autres pour de 
«bonnes raisons", et qu'alors un lieu sociofuge peut être recherché. Ses investigations empiriques dans les bibliothèques universitaires l'illustrent très clairement. Cela signifie que le caractère sociofuge de la bibliothèque est également ce qui fait la «bonne ambiance » de la bibliothèque (Sommer, 1967).

14. Pour tout ce passage : la catégorie de "populaire» devrait être empiriquement affinée, notamment par les appartenances de genre et d'âge ; il y a fort à parier que la liberté dont il est question concerne avant tout les «jeunes » " hommes » au sein de ces classes populaires. Je suis redevable à Karim Hammou de cette remarque.

15. Cela rejoint l'analyse que j'ai proposée pour la fin de Do the Right Thing (Spike Lee, 1989): quand Radio Raheem («Radio Barjot » dans la version française) se fait détruire son ghetto-blaster hurlant «Fight the Power» de Public Enemy par Sal, le propriétaire de la pizzéria où Radio Raheem vient porter avec deux amis une revendication concernant l'absence d'AfricainsAméricains sur le «mur des célébrités » de la pizzéria, mur uniquement peuplé de gloires italoaméricaines (c'est-à-dire l'origine de Sal, alors que les clients de cette pizzéria de Brooklyn sont très majoritairement noirs) (Pecqueux, 2013, p. 65-68).

16. Toute une passionnante famille de travaux traite des relations entre lien civil et lien politique, d'une politicité ordinaire, infra-politique en quelque sorte, depuis le livre pionnier en la matière de Pharo (1985) jusqu'à celui de Berger, Cefaï et Gayet-Viaud (2011). Pour une récente mise au point : Bidet, Boutet, Chave et al., 2015.

17. Pour ne citer que deux exemples en français ; il n'est pas anodin que des numéros spéciaux de revues académiques aient été consacrés à The Wire: Film Quaterly en 2008 (vol. 62 n²), Criticism en 2010 (vol. $52 n^{\circ}$ 3-4).

18. Un passage central de la série - parmi d'autres - gagnerait à être analysé à l'aune des catégories goffmaniennes : celui, à la fin de l'épisode deux de la saison trois, où le major Colvin s'adresse aux policiers du district ouest de la ville suite à la grave blessure par balle d'un de leurs collègues. Colvin y analyse l'invention fortuite $\mathrm{du}$ sac en papier brun autour des bouteilles d'alcool comme un geste de génie assurant un "compromis citoyen" entre policiers et consommateurs d'alcool dans l'espace public (les premiers perdant auparavant leur temps à poursuivre les seconds). Ce sac en papier fonctionne comme un écran de l'engagement en public : si l'engagement « masqué » reste assez transparent (il est rare que ces sacs en papier contiennent du soda), il n'en assure pas moins une légalisation d'une pratique prohibée.

19. «La zone trouve son nom à la faveur d'un malentendu significatif : tandis que les flics de base font l'analogie avec Amsterdam, 'un de ces pays où la drogue est légale', les cornerboys retranscrivent à leur manière ce mot qu'ils ne connaissent pas. En le déformant, ils font ironiquement apparaître le sens caché de l'initiative. Sous la référence à un modèle exotique de légalisation, se profile en fait une expérimentation originale dont ils seront les cobayes: Hamster-dam » (Aarons, Burdeau, Chamayou et al., op.cit., p. 67).

20. Dans la mesure où Hamsterdam n'est pas qu'une utopie et peut être rapproché des processus de gentrification, qui produisent peu ou prou les mêmes effets et les mêmes victimes.

21. Pour une lecture plus fine de cette métaphore, voir Bonicco-Donato, 2013.

\section{RÉSUMÉS}

Cet article prend la forme d'une note critique sur la récente traduction française de Behavior in Public Places d'Erving Goffman pour discuter quelques uns des liens entre la microsociologie de 
l'interaction et les études sur les ambiances. Pour cela, il retrace dans un premier temps les principaux apports du livre, notamment du point de vue de l'attention sensible et de son organisation en situation. Un deuxième temps suit le fil des pistes proposées par Goffman en direction des ambiances urbaines, comprises comme la tonalité affective dans laquelle les individus interagissent et qu'ils peuvent modifier par leurs interactions et présences. Enfin, un troisième temps s'appuie sur les séries télévisées The Wire et Treme pour illustrer et poursuivre la discussion entamée sur ambiances et civilité.

This paper is a critical review of the recent French translation of Erving Goffman's Behavior in Public Places, in order to discuss a few connections between interaction's microsociology and ambiances studies. For this purpose, it relates first the book's main contributions, especially from the attention-sensitive's point of view and its situated organisation. Secondly, the paper follows the tracks proposed by Goffman towards urban ambiances, understood as the affective tone through which individuals interact and that they can alter with their interactions and presences. Finally, the paper rests on The Wire and Treme TV series in order to illustrate and continue the discussion about ambiances and civility.

\section{INDEX}

Mots-clés : Erving Goffman, interactionnisme, ambiances, attention, engagement, civilité, The Wire

Keywords : Erving Goffman, interactionism, ambiances, attention, involvement, civility, The Wire

\section{AUTEUR}

\section{ANTHONY PECQUEUX}

Chargé de Recherche au CNRS, CRESSON (UMR AAU 1563 / Ecole Nationale Supérieure d'Architecture de Grenoble). Ses recherches portent de manière générale sur une approche écologique, sensible des expériences urbaines. Il a notamment dirigé en 2012 le numéro 90 de la revue Communications, « Les bruits de la ville »; et récemment publié « John Langshaw Austin, la perception et son ethnographie » dans Ethnographier les sens (Paul-Louis Colon éd., 2013), Paris, Petra, p. 43-70. Il est co-rédacteur en chef de la Revue Ambiances.

Page personnelle : http://www.cresson.archi.fr/EQ/EQanthony.htm anthony.pecqueux@grenoble.archi.fr 